\title{
GRB 050509b: the elusive optical/nIR/mm afterglow of a short-duration GRB ${ }^{\star}$
}

\author{
A. J. Castro-Tirado ${ }^{1}$, A. de Ugarte Postigo ${ }^{1}$, J. Gorosabel ${ }^{1}$, T. Fathkullin ${ }^{2}$, V. Sokolov ${ }^{2}$, M. Bremer ${ }^{3}$, I. Márquez ${ }^{1}$, \\ A. J. Marín ${ }^{1}$, S. Guziy ${ }^{1,4}$, M. Jelínek ${ }^{1}$, P. Kubánek ${ }^{5}$, R. Hudec ${ }^{5}$, S. Vitek ${ }^{6}$, T. J. Mateo Sanguino ${ }^{7}$, A. Eigenbrod ${ }^{8}$, \\ M. D. Pérez-Ramírez ${ }^{9}$, A. Sota ${ }^{1}$, J. Masegosa ${ }^{1}$, F. Prada ${ }^{1}$, and M. Moles ${ }^{1}$
}

\author{
1 Instituto de Astrofísica de Andalucía (IAA-CSIC), PO Box 3.004, 18.080 Granada, Spain \\ e-mail: ajct@iaa.es \\ 2 Special Astrophysical Observatory, Russian Academy of Sciences, Karanchai-Cherkessia, Nizniy-Arkhyz, 357147, Russia \\ 3 Institute de Radioastronomie Milimetrique (IRAM), 300 rue de la Piscine, 38406 Saint-Martin-d'Hères, France \\ ${ }^{4}$ Nikolaev State University, Nikolskaya 24, 54.030 Nikolaev, Ukraine \\ 5 Astronomical Institute, Academy of Sciences of the Czech Republic, 25165 Ondřejov, Czech Republic \\ ${ }^{6}$ Fakulta electrotechnická, Czech Technical University, 12135 Praha, Czech Republic \\ 7 Dept. de Ing. Electrónica, Sistemas Informáticos y Automática, Univ. de Huelva, 21.819 Palos de la Frontera (Huelva), Spain \\ 8 Laboratoire d'Astrophysique, École Polytechnique Fédérale de Lausanne, Observatoire, 1290 Sauverny, Switzerland \\ 9 Departamento de Física (EPS), Univ. de Jaén, Campus Las Lagunillas, 23071 Jaén, Spain
}

Received 27 May 2005 / Accepted 26 June 2005

\begin{abstract}
We present multiwavelength (optical/near infrared/millimetre) observations of a short duration gamma-ray burst detected by Swift (GRB 050509b) collected between 0 seconds and $\sim 18.8$ days after the event. No optical, near infrared or millimetre emission has been detected in spite of the well localised X-ray afterglow, confirming the elusiveness of the short duration events. We also discuss the possibility of the burst being located in a cluster of galaxies at $z=0.225$ or beyond. In the former case, the spectral energy distribution of the neighbouring, potential host galaxy, favours a system harbouring an evolved dominant stellar population (age $360 \mathrm{Myr}$ ), unlike most long duration GRB host galaxies observed so far, i.e. thus giving support to a compact binary merger origin. Any underlying supernova that could be associated with this particular event should have been at least 3 magnitudes fainter than the type Ib/c SN 1998bw and 2.3 mag fainter than a typical type Ia SN.
\end{abstract}

Key words. gamma rays: bursts - techniques: photometric - cosmology: observations

\section{Introduction}

Gamma Ray Bursts (GRBs) are flashes of high energy $(\sim 1 \mathrm{keV}-10 \mathrm{GeV})$ photons (Fishman \& Meegan 1995), occurring at cosmological distances. The detection of counterparts at other wavelengths for long duration GRBs, revealed their cosmological origin (see van Paradijs et al. 2000, for a review) and it is accepted nowadays that they are associated with the death of massive stars. On the other hand, the nature of short duration GRBs, a class that comprises about $25 \%$ of all events (Mazets et al. 1981; Kouveliotou et al. 1993), still remains a puzzle. No counterparts have been discovered so far, in spite of intense efforts in order to detect the optical, infrared and radio

\footnotetext{
* Based on observations taken with the $1.2 \mathrm{~m}$ Mercator telescope at Observatorio del Roque de los Muchachos, with the $1.5 \mathrm{~m}$ telescope at Observatorio de Sierra Nevada, with the $2.2 \mathrm{~m}$ and $3.5 \mathrm{~m}$ telescopes at the Centro Astronómico Hispano Alemán (CAHA) at Calar Alto (operated jointly by the Max-Planck Institut für Astronomie and the Instituto de Astrofísica de Andalucía (CSIC)) and with the $6.0 \mathrm{~m}$ telescope at the Special Astrophysical Observatory in Russia.
}

counterparts to several short, hard bursts (Kehoe et al. 2001; Gorosabel et al. 2002; Hurley et al. 2002; Klotz et al. 2003). A possible optical transient, related to GRB 000313 was proposed by Castro-Tirado et al. (2002) but a firm conclusion could not be established.

The Swift satellite (Gehrels et al. 2004) offers unique capabilities for the detection of GRBs thanks to its high sensitivity and imaging capabilities at $\gamma$-rays, X-rays and optical wavelengths. The short GRB 050509b was discovered by Swift/BAT detector on 9 May 2005. The burst started at 04:00:19.23 UT and lasted for $\approx 40 \mathrm{~ms}$, putting it in the "short-duration" class of GRBs. It had a fluence of $(0.95 \pm 0.25) \times 10^{-8} \mathrm{erg} \mathrm{cm}^{-2}$ in the $15-150 \mathrm{keV}$ range (Gehrels et al. 2005). The prompt dissemination (13.7 s) of the GRB position (Hurkett et al. 2005) enabled prompt responses of automated and robotic telescopes on ground, like ROTSE-III (Rykoff et al. 2005), RAPTOR (Wozniak et al. 2005) and BOOTES-1 (shown in this Letter), although no prompt afterglow emission was detected. By the time when Swift slewed and started data acquisition (about $60 \mathrm{~s}$ after the event onset), a fading X-ray emission was detected by 
Table 1. Journal of optical and nIR observations of the GRB 050509b field.

\begin{tabular}{|c|c|c|c|c|c|}
\hline Date of 2005 UT (mid exposure) & Telescope/instrument & Filtre & Exposure time (s) & Seeing $(\operatorname{arcsec})$ & $\overline{\text { Limiting magnitude }(3 \sigma)}$ \\
\hline May 09, 04:00.25 & BOOTES-1 (ASCCD) & $R$ & 30 & 3.0 & 6.0 \\
\hline May 09, 04:40 & 1.2Mer (MEROPE) & $R$ & 600 & 2.5 & 21.0 \\
\hline May 11, 20:00 & 6.0BTA (SCORPIO) & $R$ & 7020 & 2.0 & 26.0 \\
\hline May $18,23: 00$ & $1.5 \mathrm{OSN}(\mathrm{CCD})$ & $I$ & 3600 & 1.4 & 21.9 \\
\hline May 09, 23:22 & 3.5CAHA (OMEGA2000) & $K$ & 2730 & 1.6 & 18.8 \\
\hline May 10, 00:05 & 3.5CAHA (OMEGA2000) & $J$ & 3656 & 2.1 & 21.2 \\
\hline May 10, 02:29 & 3.5CAHA (OMEGA2000) & $H$ & 3563 & 2.4 & 20.5 \\
\hline May 10, 21:50 & 3.5CAHA (OMEGA2000) & $J$ & 1897 & 1.9 & 20.3 \\
\hline May $12,21: 55$ & 3.5CAHA (OMEGA2000) & $H$ & 2314 & 1.7 & 20.3 \\
\hline
\end{tabular}

Table 2. Journal of mm observations of the GRB 050509b field.

\begin{tabular}{|c|c|c|c|c|c|}
\hline Date of 2005 UT & Configuration & Detection level $(\mathrm{mJy}, 1 \sigma)$ & Frequency $(\mathrm{GHz})$ & Beam $(\operatorname{arcsec})$ & Position angle (deg) \\
\hline \multirow[t]{2}{*}{ May $11,02: 56-03: 38$} & $6 \mathrm{Dp}$ & 1.9 & 80.327 & $32.2 \times 4.44$ & -136 \\
\hline & & 12.0 & 242.842 & $9.89 \times 1.62$ & -137 \\
\hline \multirow[t]{2}{*}{ May $11,21: 38-23: 49$} & $6 \mathrm{Dp}$ & 0.8 & 80.467 & $9.56 \times 4.51$ & +66 \\
\hline & & 5.9 & 242.842 & $3.24 \times 1.73$ & +64 \\
\hline \multirow[t]{2}{*}{ May $13,00: 26-02: 44$} & $6 \mathrm{Dp}$ & 1.0 & 80.467 & $25.30 \times 4.54$ & +48 \\
\hline & & 4.4 & 221.501 & $6.05 \times 1.70$ & -128 \\
\hline May $16,17: 33-22: 28$ & $5 \mathrm{Dp}$ & 0.3 & 92.682 & $6.55 \times 5.44$ & +93 \\
\hline
\end{tabular}

the Swift/XRT; this can be considered as the first clear detection of an afterglow in a short duration GRB (Kennea et al. 2005). This triggered a multiwavelength campaign at many observatories aimed at detecting the afterglow at other wavelengths, as in the case of the long duration GRB class. Here we report the results of the multiwavelength observations carried out by our group, from millimetre ( $\mathrm{mm}$ ) wavelengths to the optical bands.

\section{Observations and data reduction}

The BOOTES-1 very wide field camera, located at Estación de Sondeos Atmosféricos (INTA-CEDEA) in Huelva (Spain), observed the region of the sky containing the Swift/BAT error box of GRB 050509b as part of its routine observing schedule (Castro-Tirado et al. 2004). A $30 \mathrm{~s}$ exposure started at 04:00:00 UT (19 s prior to the onset of the $40 \mathrm{~ms}$ short burst), with the following frame starting at 04:01:00 UT. A limiting (unfiltered, airmass 4.0) magnitude of 6.0 is derived for any prompt optical flash arising from this event.

ToO observations in the optical were triggered starting $0.53 \mathrm{~h}$ after the event at the $1.2 \mathrm{~m}$ Mercator telescope (+ MEROPE CCD camera) at Observatorio del Roque de los Muchachos in La Palma (Spain). Subsequently, ToO observations were made at the $1.5 \mathrm{~m}$ telescope at Observatorio de Sierra Nevada in Granada (Spain) and at the $6.0 \mathrm{~m}$ BTA telescope (+ SCORPIO) at the Special Astrophysical Observatory (SAO-RAS) in Nizhnij-Arkhyz (Russia), and at the $2.2 \mathrm{~m}$ telescope (+BUSCA) at Calar Alto (Spain). Near infrared (nIR) observations were obtained at the $3.5 \mathrm{~m}$ telescope
(+ OMEGA2000) at Calar Alto as part of the ALHAMBRA ${ }^{1}$ back-up programme. Table 1 displays the observing log.

Additionally, mm observations were obtained at the Plateau de Bure Interferometer (PdBI) as part of our ToO programme. The PdBI observed the source on May 11 and May 13 (6 antennas compact D configuration) and May 16 (5 antennas compact $\mathrm{D}$ configuration). The data reduction was done with the standard CLIC and MAPPING software distributed by the Grenoble GILDAS group ${ }^{2}$; the flux calibration is relative to the carbon star MWC349. Table 2 displays the observing log.

In order to subtract in all of our optical and nIR images the contribution of the bright elliptical galaxy 9.'5 away from the XRT error box, we modeled it using the ELLIPSE routine under IRAF ${ }^{3}$. We used the residual to perform further analysis in the optical and nIR images. The optical field was calibrated using the field photometry provided by Henden (2005). The nIR images were calibrated using the 2MASS Catalogue.

\section{Results and discussion}

The main observational result is the lack of any variable optical/nIR/mm counterpart in our images, within the refined Swift/XRT error box (Gehrels et al. 2005), in spite of the intensive searches, in agreement with the upper limits reported by ROTSE-III and RAPTOR and Swift/UVOT

\footnotetext{
1 http://alhambra.iaa.es: 8080

2 http://wWW.iram.es/IRAMFR/GS/gildas/gildas.html

3 IRAF is distributed by the NOAO, which are operated by USRA, under cooperative agreement with the US NSF.
} 
(Gehrels et al. 2005) for the prompt optical emission and by PAIRITEL (Bloom et al. 2005) for the prompt nIR emission. Similarly, the deep upper limits reported at the Keck (Bloom et al. 2005; Cenko et al. 2005) and at the VLT (Hjorth et al. 2005a; Covino et al. 2005) are in agreement with our conclusions drawn from the deep 6.0BTA observations.

\subsection{No optical/nIR/mm afterglow at all?}

The fact that the detected X-ray afterglow for GRB 050509b (Gehrels et al. 2005) is the faintest of all afterglows detected by the Swift/XRT so far may indicate that the density of the surrounding medium where the progenitor took place is much lower than the typical value of $\approx 1 \mathrm{~cm}^{-3}$ derived for several long-duration GRBs. Assuming that the X-ray afterglow is caused by synchrotron emission, as in the case of the longduration family, one should also expect some contribution at nIR and optical wavelengths, but a simple $S_{\mathrm{X} \text {-ray }} / S_{\text {optical }}$ scaling would predict prompt optical fluxes $\sim 10^{2}$ (i.e. $\sim 5 \mathrm{mag}$ ) fainter than the optical afterglows observed so far for the long-duration events. Moreover, the combined Swift/XRT and Chandra X-ray Observatory ( $C X O)$ observations (Patel et al. 2005) imply that the decay exponent $\alpha$ is 1.1 (Gehrels et al. 2005), i.e., an optical afterglow might have rapidly decayed in brightness with a similar power-law decay index for $v_{\mathrm{opt}}<v_{\mathrm{X}}<v_{\mathrm{c}}$ (Sari et al. 1998).

\subsection{Is GRB 050509b at $z=0.225$ ?}

GRB 050509b is located in the direction of the NSC J123610+285901 cluster of galaxies (Gal et al. 2003) at $z=0.225$ (see also Bloom et al. 2005). In fact, during the prompt search for the X-ray afterglow detected by Swift/XRT, $C X O$ has detected the diffuse X-ray emission from the intracluster gas rather than the point source itself (Patel et al. 2005).

If this would be the case, taking into account the proximity (9.'5) of the X-ray error box to the elliptical galaxy 2MASX J12361286+2858580 (Fig. 1), the relationship to it cannot be discarded. It would be only $\sim 33 \mathrm{kpc}$ in projection $\left(3.563 \mathrm{kpc} /{ }^{\prime \prime}\right)$, as the angular size distance $D_{\mathrm{A}}=735 \mathrm{Mpc}$, considering a Hubble constant of $H_{0}=71 \mathrm{~km} \mathrm{~s}^{-1} \mathrm{Mpc}^{-1}$, a matter density $\Omega_{\mathrm{m}}=0.3$, and a cosmological constant $\Omega_{\Lambda}=0.7$. Assuming this, the progenitor would have been originated in the halo of the elliptical galaxy and could favour a neutron-star merger origin (Goodman et al. 1987; Eichler et al. 1989; Narayan et al. 1992), a physical scenario that can explain a short-duration burst like GRB 050509b. We note that past searches for correlations between clusters of galaxies and GRBs did not reveal positive results (Hurley et al. 1997; Gorosabel \& Castro-Tirado 1997).

Radio emission in (or close to) the center of this galaxy has been detected at the WSRT (van der Horst et al. 2005) although no emission lines are seen in the optical spectrum (Bloom et al. 2005). The restframe colours (and therefore the associated K-corrections) have been obtained based on the HyperZ code (Bolzonella et al. 2000). Fitting synthetic

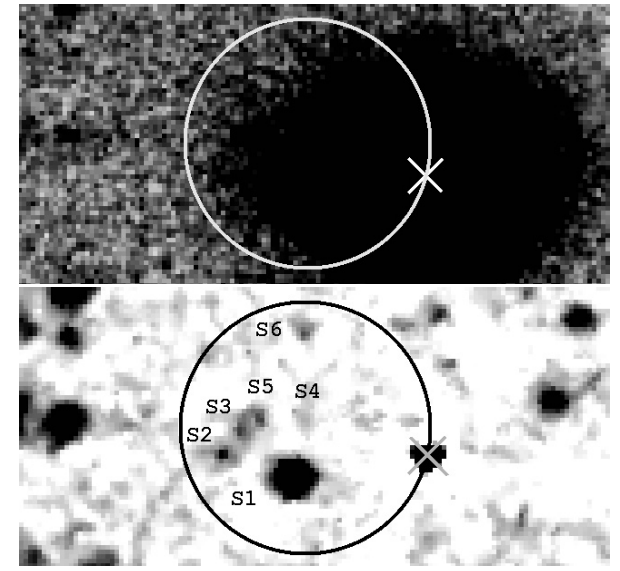

Fig. 1. The deep $R$ band image of the GRB 050509b field taken at the 6.0BTA on 11 May 2005. The contribution of the elliptical galaxy, marked with a cross (upper panel) has been removed in order to better show the content of the Swift/XRT error box (lower panel). The six sources S1-S4 (Cenko et al. 2005) and S5-S6 (Hjorth et al. 2005b) within the Swift/XRT error box are indicated. The field is $41^{\prime \prime} \times 20^{\prime \prime}$ with North up and East to the left.

spectral energy distributions (SED) templates to the $B$ band magnitude $(19.73 \pm 0.08)$, derived from the Bok telescope image (Engelbracht \& Eisenstein 2005) and from our own data and taking the nIR magnitudes from the 2MASS, produces $U-B=-0.50 \pm 0.20, B-V=1.08 \pm 0.20, V-R=0.31 \pm 0.20$ and $R-I=0.69 \pm 0.20$, (correcting all for the Galactic reddening $E(B-V)=0.019$; Schlegel et al. 1998). At $z=0.225$, this is a rather luminous galaxy, with $M_{B}=-20.6$ and $M_{R}=-22.2$. According to HyperZ, the BRIJHK band SED of the neighbour galaxy at $z=0.225$ favours $\left(\chi^{2} /\right.$ d.o.f. $\left.=2.4\right)$ a moderately extincted $\left(A_{\mathrm{v}} \sim 0.4 \mathrm{mag}\right)$ galaxy harbouring an evolved dominant stellar population (age $\sim 360 \mathrm{Myr}$ ). Figure 2 shows the SED of the elliptical galaxy.

In this scenario, an intriguing possibility arises if the event would be the result of a stellar collapse, similarly to the long duration GRBs. At such a redshift, an underlying type $\mathrm{Ib} / \mathrm{c}$ SN similar to SN 1998bw/GRB 980425 (Galama et al. 1998) should have peaked at $R \sim 21$, about 20 days since the burst onset. An underlying Type Ia SN is also expected if the event is the result of the gravitational collapse of a $\mathrm{C} / \mathrm{O}$ white dwarf into a neutron star (Dar \& De Rújula 2004). Our optical limits in the $R$-band 18.5 days after the event onset imply that the peak flux of any underlying supernova should have been $\sim 3$ mag fainter than the one observed for the type Ib/c SN 1998bw/GRB 980425 (Galama et al. 1998), and 2.3 mag fainter than a typical type Ia SN (Filippenko 1997, and references therein), in agreement with the VLT results (Hjorth et al. 2005a).

\subsection{Is GRB 050509b at high redshift?}

It is also plausible that GRB $050509 \mathrm{~b}$ has been originated at a redshift considerably higher than 0.225 , i.e. it could lie in the S1 galaxy or in one of the fainter sources (S2-S6) detected within the XRT error box (Fig. 1) at unknown redshift (see Bloom et al. 2005) or it occurred in a much more distant object, 




Fig. 2. Synthetic fit to the BRIJHK band photometric points of the elliptical galaxy. The best fist is achieved with a moderately extincted $\left(A_{\mathrm{v}}=0.4 \mathrm{mag}\right)$ galaxy harbouring an evolved stellar population (age $360 \mathrm{Myr}$ ). The SED fit assumed a Salpeter (1955) IMF and a Calzetti et al. (2000) extinction law.

at very high redshift. In the former case, extinction might have played a considerable role in order to hide optical variability in the first hours/days following the event. However, the lack of detection of a nIR transient in the observations presented here disfavours this argument. On the contrary, if GRB 050509b arose from a high-redshift host galaxy, it would have easily been beyond the limit of the optical/nIR telescopes, because of the Lyman $\alpha$ blanketing affecting the optical band.

\section{Conclusions}

We have shown multiwavelength observations of the short duration gamma-ray burst detected by Swift (GRB 050509b) between $0 \mathrm{~s}$ and $\sim 18.8$ days after the event. No optical $/ \mathrm{nIR} / \mathrm{mm}$ afterglow emission has been detected, in spite of the reported $\mathrm{X}$-ray afterglow detection by Swift few minutes after the event, confirming the elusiveness of the afterglow of the short duration events. The spectral energy distribution of the neighbouring, potential host galaxy, favours a system harbouring an evolved dominant stellar population (age $360 \mathrm{Myr}$ ), unlike most long duration GRB host galaxies observed so far, i.e. thus giving support to a compact binary merger origin. Any underlying supernova that could be associated with this particular event should have been at least 3 mag fainter than SN 1998bw and 2.3 mag fainter than a type Ia SN.

GRB 050509b is the second short duration GRB which is detected by Swift/BAT after GRB 050202 (which occurred too close to the Sun and could not be properly followed-up), and the first one localised with high accuracy by Swift/XRT. In spite of CGRO/BATSE detecting about $1 / 4$ of events belonging to the short duration class, Swift has only detected 2 (out of $\sim 40$ events), most likely due to its softer threshold energy. However, thanks to its extraordinary repointing capabilities, the accurate localisations for future events and the corresponding multiwavelength follow-up, will shed more light on the origin of the short-duration GRBs.

Acknowledgements. We acknowledge the comments from the anonymous referee, and C. W. Engelbracht and D. J. Eisenstein for making available their B-band image. This work is based partly on observations carried out with the IRAM Plateau de Bure Interferometer, supported by INSU/CNRS (France), MPG (Germany) and IGN (Spain); and on data products from the Two Micron All Sky Survey (2MASS), which is a joint project of the Univ. of Massachusetts and the IPAC/CalTech, funded by NASA and the NSF. The work was partially supported by the grant A3003206 of the Grant Agency of the Academy of Sciences of the Czech Republic, by the RFBR grant 04-02-16300a and by the Program of the Presidium of RAN entitled "Non-stationary Phenomena in Astronomy 2005" and by the Spanish MEC programmes AYA2004-01515 and ESP2002-04124-C03-01.

\section{References}

Bloom, J. S., Prochaska, J. X., Pooley, D., et al. 2005, ApJ, submitted [arXiv: astro-ph/0505480]

Bolzonella, M., Miralles, J.-M., \& Pelló, R. 2000, A\&A, 363, 476

Calzetti, D., Armus, L., Bohlin, R. C., et al. 2000, ApJ, 533, 682

Castro-Tirado, A. J., Castro Cerón, J. M., Gorosabel, J., et al. 2002, A\&A, 393, L55

Castro-Tirado, A. J., Jelínek, M., Mateo Sanguino, T. J., et al. 2004, AN, 325, 679

Cenko, S. B., Soifer, B. T., Bian, C., et al. 2005, GCN Circ., 3401

Covino, S., Israel, G. L., Antonelli, L. A., et al. 2005, GCN Circ., 3413

Dar, A., \& De Rújula, A. 2004, Phys. Rep., 405, 203

Eichler, D., Livio, M., Piran, T., \& Schramm, D. N. 1989, Nature, 340, 126

Engelbracht, C. W., \& Eisenstein, D. J. 2005, GCN Circ., 3420

Filippenko, A. V. 1997, ARA\&A, 35, 309

Fishman, G. J., \& Meegan, C. A. 1995, ARA\&A, 33, 415

Gal, R. R., de Carvalho, R. R., Lopes, P. A. A., et al. 2003, AJ, 125, 2064

Galama, T. J., Vreeswijk, P. M., van Paradijs, J., et al. 1998, Nature, 395,670

Gehrels, N., Chincarini, G., Giommi, P., et al. 2004, ApJ, 611, 1005

Gehrels, N., Barbier, L., Barthelmy, S. D., et al. 2005, Nature, submitted [arXiv: astro-ph/0505630]

Goodman, J., Dar, A., \& Nussinov, S. 1987, ApJ, 314, L7

Gorosabel, J., \& Castro-Tirado, A. J. 1997, ApJ, 483, L83

Gorosabel, J., Andersen, M. I., Hjorth, J., et al. 2002, A\&A, 383, 112

Henden, A. A. 2005, GCN Circ., 3454

Hjorth, J., Sollerman, J., Gorosabel, J., et al. 2005a, ApJ, submitted [arXiv: astro-ph/0506123]

Hjorth, J., Sollerman, J., Jensen, B. L., et al. 2005b, GCN Circ., 3410

Hurkett, C., Rol, E., Barbier, L., et al. 2005, GCN Circ., 3381

Hurley, K., Hartmann, D., Kouveliotou, C., et al. 1997, ApJ, 479, L113

Hurley, K., Berger, E., Castro-Tirado, A. J., et al. 2002, ApJ, 567, 447

Kehoe, R., Akerlof, C., Balsano, R., et al. 2001, ApJ, 554, L159

Kennea, J. A., Burrows, D. N., Housek, J., et al. 2005, GCN Circ., 3383

Klotz, A., Böer, M., \& Atteia, J.-L. 2003, A\&A, 404, 815

Kouveliotou, C., Meegan, C. A., Fishman, G. J., et al. 1993, ApJ, 413, 101

Mazets, E. P., Golenetskii, S. V., Ilyinskii, V. N., et al. 1981, Ap\&SS, 80,119

Narayan, R., Paczyński, B., \& Piran, T. 1992, ApJ, 395, L83

Patel, S., Kouveliotou, C., Burrows, D. N., et al. 2005, GCN Circ., 3419

Rykoff, E. S., Swan, H., Schaefer, B., et al. 2005, GCN Circ., 3382

Salpeter, E. E. 1955, ApJ, 121, 161

Sari, R., Piran, T., \& Narayan, R. 1998, ApJ, 497, L17

Schelgel, D. J., Finkbeiner, D. P., \& Davis, M. 1998, ApJ, 500, 525

van der Horst, A. J., Wiersema, K., Wijers, R. A. M. J., et al. 2005, GCN Circ., 3405

van Paradijs, J., Kouveliotou, C., \& Wijers, R. A. M. J. 2000, ARA\&A, 38, 379

Wozniak, P., Vestrand, W. T., Wren, J., et al. 2005, GCN Circ., 3414 